\title{
Kinetic network model to explain gain-of-function . mutations in ERK2 enzymes
}

\author{
${ }_{5}$ M. Misiura and A. B. Kolomeisky
}

\section{ABSTRACT}

ERK2 is a kinase protein that belongs to a Ras/Raf/MEK/ERK signalling pathway, which is activated in response to a range of 8 extracellular signals. Malfunctioning of this cascade leads to variety of serious diseases, including cancers. This is often caused 9 by mutations in proteins belonging to the cascade, frequently leading to abnormally high activity of the cascade even in the 10 absence of external signal. One such gain-of-function mutation in ERK2 protein, called a sevenmaker mutation (D319N), was 11 discovered in 1994 in Drosophila. This mutation leads to disruption of interactions of other proteins with D-site of ERK2 and 12 results, contrary to expectations, in increase of its activity in vivo. However, no molecular mechanism to explain this effect has ${ }_{13}$ been presented so far. The difficulty is that this mutation should equally negatively affect interactions of ERK2 with all substrates, ${ }_{14}$ activators and deactivators. In this paper, we present a quantitative kinetic network model that gives a possible explanation 15 of the increased activity of mutant ERK2 species. A simplified biochemical network for ERK2, viewed as a system of coupled ${ }_{16}$ Michaelis-Menten processes, is presented. Its dynamic properties are calculated explicitly using the method of first-passage 17 processes. The effect of mutation is associated with changes in the strength of interaction energy between the enzyme and ${ }_{18}$ the substrates. It is found that the dependence of kinetic properties of the protein on the interaction energy is non-monotonic, ${ }_{19}$ suggesting that some mutations might lead to more efficient catalytic properties, despite weakening inter-molecular interactions. ${ }_{20}$ Our theoretical predictions agree with experimental observations for the sevenmaker mutation in ERK2. It is also argued that the ${ }_{21}$ effect of mutations might depend on the concentrations of substrates.

\section{${ }_{22}$ INTRODUCTION}

23

Mitogen-activated protein (MAP) kinase ERK2 (Extra${ }_{24}$ cellular Signal-Regulated Kinase 2) is an enzyme that plays ${ }_{25}$ important role in a variety of biochemical processes. It is ${ }_{26}$ activated in response to several extracellular signals such as 27 mitogen, interleukin, growth factors and cytokines $(1,2)$, op${ }_{28}$ erating as a part of a Ras/Raf/MEK/ERK signalling pathway, ${ }_{29}$ which is crucial for cell functioning (3-5). ERK2 a small 42 3o $\mathrm{kDa}$ protein, consisting of $\mathrm{C}$-terminal and $\mathrm{N}$-terminal domains ${ }_{31}$ (6-8). It's an ATP-dependent enzyme and the ATP-binding 32 site, as well as the catalytic site, are located in the region be${ }_{3}$ tween the main domains. Unlike many enzymes, ERK2 does ${ }_{34}$ not bind its substrates in the immediate vicinity of catalytic ${ }_{35}$ site, but instead it utilizes the so-called recruiting sites (dock${ }_{36}$ ing sites), which are located 15-20 $\AA$ away from the place ${ }_{37}$ where the catalysis occurs. These binding sites are usually 38 referred to as D-recruiting site (DRS) and F-recruiting site ${ }_{39}$ (FRS), and they are responsible for recognition of multiple 40 substrates with different structures (9-19).

To become catalytically active, ERK2 requires phospho42 rylation of two of its residues: Tyr185 and Thr183 (20). ${ }_{43}$ Phosphorylation leads to alteration of mutual orientation of ${ }_{44}$ domains and their dynamics $(7,21-25)$. Activation of ERK2 45 is normally done by MAP/ERK kinases (MEK) (26). Ac46 tive ERK2, in its turn, can be deactivated by a number of 47 phosphatases $(1,20)$. Combination of these two processes 48 activation and deactivation - enables precise control of ERK2
49 activity, providing a robust and efficient method to respond to 50 external signals. Since ERK2 regulates many critically impor51 tant processes, including cell growth, cell differentiation and 52 cell proliferation, the alteration of its normal enzymatic activ${ }_{53}$ ity can lead to serious negative effects, such as uncontrollable 54 tissue growth, which was shown to be linked to a variety of 55 diseases, including cancers (27-30).

An interesting example of ERK2 malfunctioning is the ex57 istence of gain-of-function mutations inside the Ras/Raf/MEK/ERK 58 signalling pathway. Such mutations can alter the structure of 59 one of the kinases in a phosphorelay, thus preventing the ac${ }_{60}$ tivity of ERK2 from being regulated properly, and eventually ${ }_{61}$ leading to disease $(3,30)$. The most known gain-of-function 62 mutation in ERK2 (D319N) is called sevenmaker, and was it ${ }_{63}$ discovered in Drosophila in 1994 as a result of genetic screen64 ings for mutations that activate the sev signalling pathway in ${ }_{65}$ the absence of signal (31-34). The mutation is located in the ${ }_{66}$ DRS (docking site) of ERK2 in the common domain (CD) 67 region (35).

The fact that the sevenmaker mutation activates the en69 zyme is rather surprising, because it is expected that this 70 mutation should negatively influence interactions of ERK2 71 with all substrates, activators and deactivators in a similar 72 fashion. One would suggest then the mutation should lower 73 the enzymatic activity. Indeed, there are experimental obser74 vations $(35,36)$ suggesting that many substrates, activators 75 (including MEK) and deactivators, use the DRS site and, 
${ }_{76}$ in particular, the CD domain to recognize the ERK2 pro- ${ }_{128}$ constant $u$ to reach the state 1 (labelled as ERK2.M): see ${ }_{77}$ tein. They interact using a so called kinase interaction motif ${ }_{129}$ Figure 1. From the state 1, ERK2 can return back to the state 78 (KIM), which consists of 2-3 positively charged Lys and/or ${ }_{130} 0$ with a rate $w$ by dissociating from the complex with MEK, ${ }_{79}$ Arg residues (19). Thus the sevenmaker mutation should dis- ${ }_{131}$ or it can be phosphorylated to reach the state 2 (ppERK2) 80 rupt all ERK2-involving processes in the similar way, so it ${ }_{132}$ with a rate $\alpha$. After that, ppERK2 can either be dephosphory${ }_{81}$ is surprising that it can lead to apparent increase of ERK2 ${ }_{133}$ lated through the formation of a complex with a phosphatase 82 activity in vivo $(31,32)$. Despite the fundamental importance ${ }_{134}$ (state 3, ERK2-D) with the rate constant $u$, or it can remain 83 of ERK2, molecular mechanisms of its gain-of-function mu- ${ }_{135}$ active and phosphorylate its own substrates by forming the ${ }_{84}$ tations (and specifically the sevenmaker mutation) remain not ${ }_{85}$ well understood. One proposal to explain these observations ${ }_{86}$ is based on the fact that there are only two ERK2 activators, ${ }_{87}$ MEK1 and MEK2, while there are many deactivators. It was 88 suggested that some deactivators might be less affected by the 89 disrupted interaction with the CD domain (35). To support 90 this, there are experimental data showing that the mutation ${ }_{91}$ D319N in the ERK2 is less sensitive to dephosphorylation ${ }_{92}(37,38)$. However, that does not resolve the problem entirely 9з since the ability of ERK2 to phosphorylate substrates should ${ }_{94}$ be also reduced by comparable amount (35).

${ }_{136}$ substrate-enzyme complex with the rate constant $u$ (state 4, ${ }_{137} \mathrm{ppERK} 2 \cdot \mathrm{S}$ ), and producing the product (state 5, ppERK2+P) ${ }_{138}$ with the rate $\alpha$. To simplify calculations, in this model we ${ }_{139}$ assume that the corresponding rate constants in all Michaelis${ }_{140}$ Menten reactions for different processes are equal to each ${ }_{141}$ other so that there are only three kinetic parameters in the 142 system: $u, w$ and $\alpha$. This assumption is based on the fact that ${ }_{143}$ all enzymatic processes are taking place at the same location 144 and they involve chemical species that are not very dissimilar. ${ }_{145}$ But it is also important to notice that the relaxing of this ${ }_{146}$ condition (making all corresponding rates different) will not ${ }_{95} \quad$ In this paper, we propose a theoretical model that quanti${ }_{96}$ tatively explains the effect of the sevenmaker mutation. It is ${ }_{97}$ based on the kinetic network description of the system with the 98 additional assumption that the mutation equally changes inter99 action energies between ERK2 and all substrates, activators 100 and deactivators. By analyzing a simplified regulation network ${ }_{101}$ of ERK2, built as a system several coupled Michaelis-Menten 102 processes, the kinetic properties of ERK2 proteins are evalu${ }_{103}$ ated explicitly via a first-passage method. It is shown that the ${ }_{104}$ effective chemical kinetic properties in these systems might ${ }_{105}$ change non-monotonically as a function of the interactions. ${ }_{106}$ This suggests that some mutations might lead to more efficient ${ }_{107}$ catalytic properties of ERK2 protein variants, despite the ${ }_{108}$ decrease of the interaction energies. It is argued that this is a ${ }_{109}$ possible molecular mechanism of gain-of-function mutations 110 in ERK2, explaining the experimental observations on the 111 sevenmaker mutation.

\section{${ }_{112}$ METHODS}

\section{${ }_{113}$ Kinetic Network Model}

114

115

[Figure 1 about here; moved to end of manuscript by endfloat.]

116 To clarify the molecular mechanisms of increased activity ${ }_{117}$ for the sevenmaker mutation, one should analyze the kinetic ${ }_{118}$ properties of the biochemical regulation pathway of ERK2. ${ }_{119}$ Although it is known that ERK2 functioning involves many ${ }_{120}$ biochemical states and transitions, we consider a minimal ${ }_{121}$ simplified version of the regulation scheme as presented in ${ }_{122}$ Figure 1. Our idea is to approximate the regulation network ${ }_{123}$ as three coupled Michaelis-Menten processes that correspond 124 to main processes involving this enzyme: activation, inacti${ }_{125}$ vation and ERK2-mediated phosphorylation (1). In the state ${ }_{126} 0$ (labelled as ERK2) the enzyme molecule is inactive (not ${ }_{127}$ phosphorylated) and it can bind MEK enzyme with a rate
${ }_{147}$ qualitatively change the main theoretical predictions of this

${ }_{148}$ work while it will make the mathematical calculations much 149 more complicated.

In evaluating the catalytic properties of this system, we ${ }_{151}$ employ a method of first-passage processes that was success152 fully utilized for analyzing multiple processes in Chemistry, ${ }_{153}$ Physics and Biology $(39,40)$. The idea is to introduce a first${ }_{154}$ passage probability density function $F_{n}(t)$, which is defined 155 as a probability to complete the reaction (i.e., to reach the ${ }_{156}$ final state 5) at time $t$ if at $t=0$ the system was in the state $n$. ${ }_{157}$ Determining these functions will provide a full dynamic de${ }_{158}$ scription of the catalytic process in this system. The temporal 159 evolution of first-passage probabilities is governed by a set 160 of the backward master equations $(39,40)$, which are closely ${ }_{161}$ related to standard chemical kinetics equations:

$$
\frac{d F_{0}(t)}{d t}=u_{M} F_{1}(t)-u_{M} F_{0}(t)
$$

162

$$
\frac{d F_{1}(t)}{d t}=\alpha F_{2}(t)+w F_{0}(t)-(w+\alpha) F_{1}(t) ;
$$

163

$$
\begin{gathered}
\frac{d F_{2}(t)}{d t}=u_{D} F_{3}(t)+u_{S} F_{4}(t)-\left(u_{D}+u_{S}\right) F_{2}(t) ; \\
\frac{d F_{3}(t)}{d t}=\alpha F_{0}(t)+w F_{2}(t)-(w+\alpha) F_{3}(t) ; \\
\frac{d F_{4}(t)}{d t}=\alpha F_{5}(t)+w F_{2}(t)-(w+\alpha) F_{4}(t) .
\end{gathered}
$$

${ }_{166}$ In these equations, we take into account the fact that the asso${ }_{167}$ ciation transition rates are proportional to the concentrations ${ }_{168}$ of participants, i.e.

$$
u_{X}=u X,
$$

${ }_{169}$ where $X=M, D$ or $S$ are the concentrations of activator, 170 deactivator and substrate, respectively. In addition, the initial 
171 condition requires that $F_{5}(t)=\delta(t)$, which means that if 172 the system starts in the state 5 the reaction is accomplished 173 immediately.

174 To calculate the first-passage probabilities, we utilize ${ }_{175}$ Laplace transformations, $\int_{0}^{\infty} e^{-s t} F_{n}(t) d t \equiv \widetilde{F_{n}}(s)$. Then Eqs. 176 (1)-(5) can be rewritten as simpler algebraic expressions:

$$
\left(s+u_{M}\right) \widetilde{F}_{0}=u_{M} \widetilde{F}_{1}
$$

$$
(s+\alpha+w) \widetilde{F_{1}}=\alpha \widetilde{F_{2}}+w \widetilde{F}_{0}
$$

$$
\left(s+u_{S}+u_{D}\right) \widetilde{F}_{2}=u_{D} \widetilde{F}_{3}+u_{S} \widetilde{F}_{4}
$$

$$
(s+\alpha+w) \widetilde{F}_{3}=\alpha \widetilde{F}_{0}+w \widetilde{F}_{2}
$$

$$
(s+\alpha+w) \widetilde{F}_{4}=\alpha \widetilde{F}_{5}+w \widetilde{F}_{2} .
$$

${ }_{181}$ The initial condition also leads to $\widetilde{F}_{5}(s)=1$. This system of 182 equations can be easily solved. Specifically, for starting the ${ }_{183}$ process in the state 0 we obtain,

$$
\widetilde{F}_{0}(s)=\frac{\alpha^{2} u^{2} M S}{\mathrm{~A}+\mathrm{B}+\Gamma+\Delta},
$$

184 where new parameters are defined as

$$
\mathrm{A}=\alpha^{2}[u s D+(u M+s)(s+u S)] ;
$$

185

$$
\mathrm{B}=s^{2}(u M+s+w)(u D+s+u S+w)
$$

$$
\Gamma=\alpha s\left[2 u^{2} M S+2 s(s+w)\right]
$$

$$
\Delta=u(2 s+w)(M+S)+u D(2 u M+2 s+w) .
$$

The explicit expressions for the first-passage probability 189 functions provide a direct way of describing all dynamic 190 properties in the system. For example, the average time to 191 reach the product state 5 starting from the state 0 , which is 192 the same as the mean time for the catalytic reaction (turnover 224 and 193 time) is given by $(39,40)$

$$
T_{0} \equiv-\frac{d \widetilde{F}_{0}}{d s}(s=0),
$$

194 from which using Eqs. 12-16 we get

$$
T_{0}=\frac{2 u M(S+D)+(\alpha+w)(D+M+S)}{\alpha u M S} .
$$

195 This result can be better understood if we rewrite it in the 196 Michaelis-Menten-like form with respect to the substrate $S$ 197 transformation $\left(T_{0}=1 / k_{\text {cat }}+K_{M} / k_{\text {cat }} * 1 / S\right)$ as follows,

$$
T_{0}=\frac{2 u M+\alpha+w}{\alpha u M}+\frac{(M+D)(\alpha+w)+2 u M D}{\alpha u M} \frac{1}{S},
$$

${ }_{98}$ from which the overall effective Michaelis-Menten parame199 ters for the kinetic network are determined in terms of the 200 microscopic transition rates:

$$
\begin{gathered}
K_{M}=\frac{(\alpha+w)(M+D)+2 u M D}{\alpha+w+2 u M} ; \\
k_{c a t}=\frac{\alpha u M}{\alpha+w+2 u M} ;
\end{gathered}
$$

202 and

$$
\frac{k_{c a t}}{K_{M}}=\frac{\alpha u M}{(\alpha+w)(M+D)+2 u M D} .
$$

203 To quantitatively analyze the effect of mutations, we as204 sume that mutations change the strength of the interactions 205 in the ERK2 complexes with activators, deactivators or sub206 strates, respectively. We define a binding energy $\epsilon$ as a measure ${ }_{207}$ of strength of such interaction. The sign is chosen so that more 208 negative values of $\epsilon$ correspond to stronger binding. Then 209 the detailed balance-like arguments allow us to estimate the 210 relations between the rate constants and the binding energy:

$$
\begin{gathered}
\frac{u}{w}=\frac{u_{0}}{w_{0}} e^{-\beta \epsilon} ; \\
\alpha=\alpha_{0} e^{\beta \epsilon} .
\end{gathered}
$$

${ }_{212}$ Here the rates with superscript 0 correspond to transition rates ${ }_{213}$ for the hypothetical situations when the interactions energies 214 are equal to zero. These equations can be understood in the 215 following way. The stronger the binding interactions, the faster 216 the system will go into the states with the complex formation 217 (states 1, 3 and 4), and the slower it will leave these states. ${ }_{218}$ Correspondingly, weaker interactions stimulate the system to ${ }_{219}$ preferentially break these complexes faster than to form them. 220 Determining the enzymatic properties of the system re${ }_{221}$ quires explicit expressions for rates that include the effect of 222 the interactions. Then we can rewrite the expressions for the ${ }_{223}$ transition rates as $(40)$ :

$$
\begin{gathered}
u=u_{0} e^{-\beta \theta \epsilon} ; \\
w=w_{0} e^{\beta(1-\theta) \epsilon},
\end{gathered}
$$

${ }_{225}$ with $\beta=1 / k_{B} T$. The parameter $0 \leq \theta \leq 1$ specifies how ${ }_{227}$ backward transitions to form or to break the complex state 228 (40). For simplicity, in the following expressions we omit the 229 subscript 0 , so that $u, w$ and $\alpha$ now replace $u_{0}, w_{0}$ and $\alpha_{0}$, 230 respectively. With these assumptions, our final equations for ${ }_{231}$ the kinetic parameters are given by:

$$
K_{M}=\frac{\left(\alpha e^{\beta \epsilon}+w e^{\beta(1-\theta) \epsilon}\right)(M+D)+2 u e^{-\beta \theta \epsilon} M D}{\alpha e^{\beta \epsilon}+w e^{\beta(1-\theta) \epsilon}+2 u e^{-\beta \theta \epsilon} M} ;
$$

$$
k_{c a t}=\frac{\alpha u e^{\beta(1-\theta) \epsilon} M}{\alpha e^{\beta \epsilon}+w e^{\beta(1-\theta) \epsilon}+2 u e^{-\beta \theta \epsilon} M}
$$


M Misiura and AB Kolomeisky

233

$$
\frac{k_{c a t}}{K_{M}}=\frac{\alpha u e^{\beta(1-\theta) \epsilon} M}{\left(\alpha e^{\beta \epsilon}+w e^{\beta(1-\theta) \epsilon}\right)(M+D)+2 u e^{-\beta \theta \epsilon} M D} .
$$

${ }_{234}$ The main advantage of this theoretical approach is that 235 now the effect of mutations can be investigated quantita236 tively because in our language it corresponds to varying the 237 interaction energy $\epsilon$.

\section{${ }_{238}$ Analysis for Michaelis-Menten Model}

239

To understand better the mechanisms of the ERK2 reg240 ulation that couple together several enzymatic processes, it ${ }_{241}$ should be compared with the simplest situation that involve 242 only a single enzymatic process. For this purpose, we present ${ }_{243}$ here a brief derivation of catalytic properties for a classical ${ }_{244}$ Michaelis-Menten kinetic scheme:

$$
\mathrm{E}+\mathrm{S} \underset{w}{\stackrel{u}{\rightleftharpoons}} \mathrm{ES} \stackrel{\alpha}{\longrightarrow} \mathrm{E}+\mathrm{P} .
$$

${ }_{245}$ The derivation follows exactly the same steps as was described ${ }_{246}$ for the model in Figure 1 above, and only main steps are ${ }_{247}$ presented. We assume here that $E+S$ corresponds to the 248 state $0, E S$ describes the state 1 , and $E+P$ is the final state 2492 . The temporal evolution of the corresponding first-passage 250 probability functions follows from

$$
\frac{d F_{0}(t)}{d t}=u_{S} F_{1}(t)-u_{S} F_{0}(t)
$$

251 and

$$
\frac{d F_{1}(t)}{d t}=\alpha F_{2}(t)+w F_{0}(t)-(w+\alpha) F_{1}(t)
$$

${ }_{252}$ After the Laplace transformation, these equations can be 253 rewritten as follows:

$$
\left(s+u_{S}\right) \widetilde{F_{0}}=u_{S} \widetilde{F}_{1}
$$

254

$$
(s+\alpha+w) \widetilde{F}_{1}=\alpha \widetilde{F}_{2}+w \widetilde{F}_{0} .
$$

255 Solving this system of equations, yields the following expres${ }_{256}$ sion for the turnover time $T_{0}$ :

$$
T_{0}=\frac{1}{\alpha}+\frac{w+\alpha}{u \alpha} \frac{1}{S}
$$

${ }_{257}$ Finally, the Michaelis-Menten parameters are given by

$$
\begin{aligned}
& K_{M}=\frac{\alpha+w}{u} ; \\
& 258 \\
& k_{\text {cat }}=\alpha \text {; } \\
& \frac{k_{c a t}}{K_{M}}=\frac{\alpha u}{\alpha+w},
\end{aligned}
$$

${ }_{260}$ where $u, w$ and $\alpha$ depend on the substrate binding energy 261 exactly as described above [see Equations 24 - 26].

262 Unless stated otherwise, the following parameters are ${ }_{263}$ utilized for calculations in the Results and Discussion section: ${ }_{264} \theta=0.5, \alpha=w=100 s^{-1}, k=10,000 s^{-1} M^{-1}, D=M=$ ${ }_{265} S=0.001 M$. These parameters are chosen just to illustrate 266 our theoretical method.

\section{RESULTS AND DISCUSSION}

268

26

270 271 272

Our main idea is that the mutations modify the interaction 273 energy between the enzyme and the substrate molecules, and 274 this leads to changes in the chemical kinetic properties of the 275 system. Using explicit expressions derived in the previous 276 section, we can analyze how the enzymatic parameters for ${ }_{277}$ ERK2 and simple Michaelis-Menten (MM) schemes vary with 278 the binding energy. The results are presented in Figures 2 and 3. 279 One can see that the enzymatic properties of ERK2 regulation 280 network differ significantly from the classical MM scheme. ${ }_{281}$ Lowering the strength of binding interactions (making $\epsilon$ more ${ }_{282}$ positive) strongly increases the catalytic rate $k_{\text {cat }}$ in the MM ${ }_{283}$ system, while the dependence of $k_{c a t}$ on $\epsilon$ is non-monotonic 284 for the ERK2 system (see Figure 2). It can be shown also that 285 in this case the highest value of $k_{c a t}$ is achieved for

$$
\frac{\epsilon_{\max }}{k_{B} T}=-\frac{\ln \left[\frac{\alpha \cdot \theta}{2 \cdot u M}\right]}{(1+\theta)} .
$$

Varying the interaction energy also leads to different ${ }_{287}$ curves for the Michaelis constant for the simple MM and for the 288 ERK2 regulation network (Figure 3). $K_{M}$ strongly increases ${ }_{289}$ with $\epsilon$ in the MM case, while for the ERK2 system $K_{M}$ is 290 slowly changing between two limiting behaviors. For very 291 strong attractive interactions $(\epsilon \rightarrow-\infty)$, we have $K_{M} \simeq D$, 292 while for strong repulsive interactions $(\epsilon \rightarrow+\infty) K_{M} \simeq$ ${ }_{293} D+M$.

294 To quantify better the enzymatic efficiency of ERK2 pro295 teins, it is more useful to consider a ratio $k_{\text {cat }} / K_{M}$, which is 296 known as a specificity constant. The larger this parameter, the 297 more efficient is enzymatic process. Figure 4 presents speci298 ficity constants as functions of the binding energies for both 299 schemes, and again the classical MM behavior is strikingly 300 different from the predictions for the ERK2 regulation system.

(36) ${ }_{301}$ The specificity constant for the MM process decreases mono302 tonically with the interaction energy, while the non-monotonic ${ }_{303}$ dependence is observed for the ERK2 case. The position of 304 the maximum here is

$$
\frac{\epsilon_{\max }}{k_{B} T}=-\frac{\ln \left[\frac{(D+M) \alpha \cdot \theta}{2 \cdot k \cdot D \cdot M}\right]}{(1+\theta)}
$$




$$
306
$$

${ }_{306}$ ing the appearance of gain-of-function mutations in the ERK2 307 system. If one assumes that the binding energy in the WT ${ }_{308}$ enzyme $\left(\epsilon_{W T}\right)$ is negative and it does not correspond to $\epsilon_{\max }$ ${ }_{309}\left(\epsilon_{W T}<\epsilon_{\max }\right)$, then mutations that change the interaction ${ }_{310}$ energies to the range between $\epsilon_{W T}$ and $\epsilon_{\max }$ will increase ${ }_{311}$ the activity of enzyme: the region between two vertical lines ${ }_{312}$ on Figure 4. In this situation, the mutation that weakens the ${ }_{313}$ interactions with the substrate will effectively make the ERK2 ${ }_{314}$ regulation network more efficient in comparison with the wild 315 type case. This might be a possible molecular mechanism of ${ }_{316}$ how the sevenmaker mutation operates in the ERK2 system. ${ }_{317}$ It is also important to note that since ERK2 is a regulatory ${ }_{318}$ enzyme it is likely to operate in vivo at low concentrations in ${ }_{319}$ the regime where the specificity constant is the main property 320 that determines the catalytic efficacy.

${ }_{321}$ The effect of gain-of-function mutations can be also ex${ }_{322}$ plained using the fluxes along the different branches of the ${ }_{323}$ regulation scheme presented in Figure 1. The flux that starts ${ }_{324}$ in the state 0 reaches the state 2 via the activation branch $\left(J_{a}\right)$, ${ }_{325}$ where it divides into the flux that goes to the final product ${ }_{326}$ via the phosphorylation branch $\left(J_{p}\right)$ and the flux that returns ${ }_{327}$ back to the state 0 via the deactivation branch $\left(J_{d}\right)$. In the ${ }_{328}$ stationary state, the flux balance requires that

$$
J_{a}=J_{d}+J_{p}
$$

${ }_{329}$ The overall enzymatic activity can be correlated with the prod- ${ }_{381}$ ззо uct formation flux $J_{p}$. Then our theoretical picture suggests ${ }_{331}$ that the sevenmaker mutation lowers both $J_{a}$ and $J_{d}$ fluxes, 332 but it decreases the deactivation flux more so that the product ${ }_{333}$ formation flux $J_{p}$ in the case of mutation is larger in compari${ }_{334}$ son with the WT ERK2 molecule, i.e., $J_{p}$ (mutant) $>J_{p}(W T)$. ${ }_{335}$ The results presented in Figure 5, where the effect of varying 336 the deactivation flux is investigated, support these arguments. ${ }_{337}$ Lowering the concentration of deactivator $(D)$ decreases the ${ }_{3 з 8}$ possibility for the system to go into the deactivation branch. ${ }_{339}$ For low $D$ the enzymatic properties of the ERK2 regulation ${ }_{340}$ pathway, as expected, approach the simple MM scheme, and ${ }_{341}$ the non-monotonic behavior as well as the ability to increase ${ }_{342}$ the enzyme's activity by mutation disappear. Only when there ${ }_{343}$ are significant fluxes via the deactivation path the gain-of344 function mutations might appear in such systems. Thus, the ${ }_{345}$ gain-of-function mutation in the ERK2 regulation network ${ }_{346}$ is the result of coupling of several enzymatic processes that ${ }_{347}$ work in opposite directions.

${ }_{348}$ Theoretical calculations presented in Figure 5 also lead to ${ }_{349}$ a surprisinng prediction that the sign of the mutation effect ${ }_{350}$ (positive gain-of-function, or negative loss-of-function) can ${ }_{351}$ be reversed by changing the concentrations of the network ${ }_{352}$ components (activators, deactivators and substrates). If one ${ }_{353}$ assumes that the sevenmaker mutation operates in the range of ${ }_{354}$ interaction strengths as given in Figure 4 (between two vertical 355 lines), then for very low concentrations of deactivators this ${ }_{356}$ mutation will no longer be increasing the enzymatic activity.
${ }_{357}$ Because ERK2 has multiple substrates, we speculate that this 358 ability of the network to selectively affect the efficiency of 359 enzymatic processes might be an additional level of regulation 360 that can benefit cellular functioning.

\section{[Figure 4 about here; moved to end of manuscript by endfloat.]}

Our theoretical views can be further supported by an${ }_{364}$ alyzing the turnover times as a function of the interaction 365 energies, as illustrated in the Figure 6. One can see that the 366 effective overall catalytic rate (inverse turnover times) shows ${ }_{367}$ the non-monotonic behavior for both the simple MM and the ${ }_{68}$ ERK2 regulation pathways. But there is a range of interaction ${ }_{69}$ energies where the increase in the binding energy lowers 370 the rate of the MM process, while the process in the ERK2 ${ }_{371}$ regulation network can go faster. This is an addition argument 372 to explain the existence of the gain-of-function mutations and 37з specifically effect of the sevenmaker mutation in ERK2. Even 374 if the mutation lowers the rate along each enzymatic pathway, 375 it might effectively increase the overall rate in the complex ${ }_{376}$ ERK2 scheme that combines all of them.

\section{[Figure 5 about here; moved to end of manuscript by endfloat.]}

[Figure 6 about here; moved to end of manuscript by endfloat.]

Because our theoretical approach makes quantitative pre32 dictions, it is important to compare them with experimental 383 observations. However, experimental data on sevenmaker mu${ }_{384}$ tation are pretty scarce, mostly qualitative and obtained using 385 very different techniques and under different experimental con386 ditions. This prevents us from explicitly including them into 387 our analysis. But we notice that Camps et al. (41) found that 388 about one order of magnitude higher concentrations of MKP-3 ${ }_{389}$ are needed in order to deactivate the mutated $E R K 2^{D 319 N}$ 390 protein variant as compared with the wild type $E R K 2$. In 391 addition, the decrease of deactivation activity by other phos392 phatases (PAC1, MKP-1 and MKP-2, approximately from 3 393 to 7 times lower) for mutated ERK2 species was reported by ${ }_{394}$ Y. Chu et al. (37). All these observations are consistent with ${ }_{395}$ our theoretical flux arguments. Furthermore, experimental 396 data by T. Tanoue et al. (35) show that the activation of ERK2 397 by MEK1 is less sensitive to sevenmaker mutation: MEK1398 facilitated activation activity of mutated $E R K 2$ is only 0.88 399 of that for WT $E R K 2$. However phosphorylation activity of ${ }_{400} E R K 2$ towards substrate MNK1 is strongly affected by the mu401 tation: phosphorylation activity of mutant $E R K 2$ is estimated 402 of being 0.11 of that of the WT enzyme. This suggests that ${ }_{403}$ all the processes involving ERK2 can be negatively affected 404 by sevenmaker mutation to a different degree. It also means 405 that the overall balance of these effects in vivo is difficult to 6 to explicitly estimate since there are many known activators, ${ }_{407}$ deactivators and substrates of ERK2 (35), and likely many со more will be discovered in the future. 
M Misiura and AB Kolomeisky

\section{${ }_{409}$ CONCLUSIONS}

${ }_{410} \quad$ Here we developed a kinetic network model to explain ${ }_{411}$ the observations of the increased enzymatic activity in the ${ }_{412}$ enzymes with the sevenmaker mutation and for other simi${ }_{413}$ lar gain-of-function mutations in the ERK2 enzymes. Our ${ }_{414}$ approach presents a comprehensive quantitative description 415 of the enzymatic properties of the wild-type and mutated ${ }_{416}$ ERK2 regulation systems. First, we constructed a simplified ${ }_{417}$ regulation network for ERK2 by arguing that it can be viewed ${ }_{418}$ as three coupled Michaelis-Menten processes that describe ${ }_{419}$ three main enzymatic processes: activation, deactivation and ${ }_{420}$ the phosphorylation. The corresponding kinetic scheme is ${ }_{421}$ analyzed then explicitly using the method of first-passage pro${ }_{422}$ cesses to evaluate the enzymatic properties of the system in ${ }_{423}$ terms of the individual transition rates and the binding energy 424 between the enzyme and the substrates. The obtained ana${ }_{425}$ lytical results are also compared with the predictions for the ${ }_{226}$ simple Michaelis-Menten scheme. It is argued that mutations ${ }_{427}$ modify the interaction energies, and this leads to changes ${ }_{428}$ in the enzymatic features of the mutant ERK2 molecules. ${ }_{429}$ Our calculations show that the catalytic properties of ERK2 ${ }_{430}$ differ significantly from the results for the simplest Michaelis-

${ }_{431}$ Menten process. We found a non-monotonic dependence of ${ }_{432}$ the specificity constant, which is a quantitative measure of ${ }_{433}$ the enzymatic efficiency, as a function of the interaction en${ }_{434}$ ergy. This suggests that some mutations might increase the ${ }_{435}$ activity of the enzyme by changing the interaction energies 484 ${ }_{436}$ to the values closer to the observed maximum. The proposed ${ }_{485}$ ${ }_{437}$ mechanism is also discussed in terms of the fluxes via dif${ }_{438}$ ferent branches of the regulation network, and theoretical ${ }_{439}$ calculations generally support it. Thus, our main conclusion 440 is that the sevenmaker mutation modifies the binding inter${ }_{441}$ action energy in such way that the deactivation process is ${ }_{442}$ affected less than the activation processes, leading to the ${ }_{443}$ effective increase in the overall catalytic activity. While the ${ }_{444}$ mutation lower the rate for each enzymatic branch for some ${ }_{445}$ interactions energies, the overall turnover time might at the ${ }_{446}$ same time decrease, making them catalytically more active. ${ }_{447}$ These theoretical predictions agree with known experimental ${ }_{448}$ observations. In addition, it was suggested that the effect of ${ }_{449}$ mutation (positive or negative) might depend on the concen${ }_{450}$ tration of activator, deactivator and substrate molecules that ${ }_{451}$ participate in the ERK2 regulation network.

${ }_{452}$ Our theoretical model provides a consistent chemical de- ${ }^{499}$ ${ }_{453}$ scription on the possible mechanisms for the gain-of-function 500 454 mutations in ERK2, giving a fully quantitative measure of ${ }^{501}$ 455 mutations, which can be in principle experimentally mea${ }_{456}$ sured. However, it is important to discuss the limitations of ${ }_{457}$ the proposed theoretical method. A weak side of our approach 458 is that a very complex biochemical network with multiple 459 states and transitions, which controls the activities of ERK2 505 460 enzymes, is simplified into a network with only three coupled 506 ${ }_{461}$ Michaelis-Menten processes. It is also assumed that the reac- ${ }_{507}$ ${ }_{462}$ tion constants for activation, deactivation and phosphorylation ${ }_{508}$
${ }_{463}$ are the same while they might differ significantly. In addition, ${ }_{464}$ current experiments give only very qualitative measurements 465 of the increase enzymatic activities of the mutant ERK2 466 molecules. But our hope is that the presented quantitative ${ }_{467}$ model will stimulate experimental and theoretical studies that 468 will test our ideas, thus advancing our understanding on the 469 mechanisms of functioning of the ERK2 as well as other 470 regulating enzymatic systems.

\section{AUTHOR CONTRIBUTIONS}

A.B.K. designed the research. A.B.K. and M.M. carried 73 out the research, analyzed the data and wrote the paper.

\section{${ }_{74}$ ACKNOWLEDGMENTS}

This work is supported by Center for Theoretical Biologi46 cal Physics NSF Grant PHY-1427654. A.B.K. also acknowl47 edges the support from Welch Foundation (Grant C-1559) 78 and from the NSF (Grant CHE-1664218).

\section{REFERENCES}

1. Futran, A. S., A. J. Link, R. Seger, and S. Y. Shvartsman, 2013. ERK as a model for systems biology of enzyme kinetics in cells. Curr. Biol. 23:R972-R979.

2. Lavoie, H., and M. Therrien, 2015. Regulation of RAF protein kinases in ERK signalling. Nat. Rev. Mol. Cell Biol. 16:281-298.

3. McCubrey, J. A., L. S. Steelman, W. H. Chappell, S. L. Abrams, G. Montalto, M. Cervello, F. Nicoletti, P. Fagone, G. Malaponte, M. C. Mazzarino, S. Candido, M. Libra, J. Bäsecke, S. Mijatovic, D. Maksimovic-Ivanic, M. Milella, A. Tafuri, L. Cocco, C. Evangelisti, F. Chiarini, and A. M. Martelli, 2012. Mutations and deregulation of Ras/Raf/MEK/ERK and PI3K/PTEN/Akt/mTOR cascades which alter therapy response. Oncotarget 3:954.

4. Cobb, M. H., and E. J. Goldsmith, 1995. How MAP kinases are regulated. J. Biol. Chem. 270:14843-14846.

5. Kim, Y., Z. Paroush, K. Nairz, E. Hafen, G. Jiménez, and S. Y. Shvartsman, 2011. Substrate-dependent control of MAPK phosphorylation in vivo. Mol. Syst. Biol. 7:467.

6. Zhang, F., A. Strand, D. Robbins, M. H. Cobb, and E. J. Goldsmith, 1994. Atomic structure of the MAP kinase ERK2 at 2.3 Å resolution. Nature 367:704.

7. Canagarajah, B. J., A. Khokhlatchev, M. H. Cobb, and E. J. Goldsmith, 1997. 1 mechanism of the MAP kinase ERK2 by dual phosphorylation. Cell 90:859-869.

8. Zhang, J., P. Shapiro, and E. Pozharski, 2012. Structure of extracellular signal-regulated kinase 2 in complex with ATP and ADP. Acta Crystallogr., Sect. F: Struct. Biol. Cryst. Commun. 68:1434-1439. 

9. Seidel, J. J., and B. J. Graves, 2001. An ERK2 docking
site in the Pointed domain distinguishes a subset of ETS
transcription factors. Genes Dev. 16:127-137.

10. Garai, Á., A. Zeke, G. Gógl, I. Törô, F. Fördôs, H. Blankenburg, T. Bárkai, J. Varga, A. Alexa, D. Emig, M. Albrecht, and A. Reményi, 2012. Specificity of linear ${ }_{563}$ motifs that bind to a common mitogen-activated protein ${ }_{564}$ kinase docking groove. Sci. Signal. 5:ra74-ra74.

11. Sheridan, D. L., Y. Kong, S. A. Parker, K. N. Dalby, and B. E. Turk, 2008. Substrate discrimination among mitogen-activated protein kinases through distinct dock- ${ }_{568}$ ing sequence motifs. J. Biol. Chem. 283:19511-19520.

12. Jacobs, D., D. Glossip, H. Xing, A. J. Muslin, and K. Kornfeld, 1999. Multiple docking sites on substrate proteins form a modular system that mediates recognition by ERK MAP kinase. Genes \& development 13:163-175.

13. Lee, S., M. Warthaka, C. Yan, T. S. Kaoud, P. Ren, and K. N. Dalby, 2011. Examining docking interactions on ERK2 with modular peptide substrates. Biochemistry 50:9500-9510.

14. Abramczyk, O., M. A. Rainey, R. Barnes, L. Martin, and K. N. Dalby, 2007. Expanding the repertoire of an ERK2 recruitment site: cysteine footprinting identifies the D-recruitment site as a mediator of Ets-1 binding. Biochemistry 46:9174-9186.

15. Slupsky, C. M., L. N. Gentile, L. W. Donaldson, C. D. Mackereth, J. J. Seidel, B. J. Graves, and L. P. McIntosh, 1998. Structure of the Ets-1 pointed domain and mitogenactivated protein kinase phosphorylation site. Proc. Natl. Acad. Sci. U. S. A. 95:12129-12134.

16. Sharrocks, A. D., S.-H. Yang, and A. Galanis, 2000. Docking domains and substrate-specificity determination ${ }^{58}$ for MAP kinases. Trends Biochem. Sci. 25:448-453.

17. Carlson, S. M., C. R. Chouinard, A. Labadorf, C. J. Lam, K. Schmelzle, E. Fraenkel, and F. M. White, 2011. ${ }^{59}$ Large-scale discovery of ERK2 substrates identifies ERK- ${ }^{592}$ mediated transcriptional regulation by ETV3. Sci. Signal. ${ }^{593}$ 4:rs11-rs11.

18. Yoon, S., and R. Seger, 2006. The extracellular signal- ${ }^{595}$ regulated kinase: multiple substrates regulate diverse ${ }^{596}$ cellular functions. Growth factors 24:21-44.

19. Zhou, B., L. Wu, K. Shen, J. Zhang, D. S. Lawrence, and 59831 Z.-Y. Zhang, 2001. Multiple regions of MAP kinase phos- 599 phatase 3 are involved in its recognition and activation 600 by ERK2. J. Biol. Chem. 276:6506-6515.

. Anderson, N. G., J. L. Maller, N. K. Tonks, and T. W. Sturgill, 1990. Requirement for integration of signals from two distinct phosphorylation pathways for activation 604 of MAP kinase. Nature 343:651.
58 21. Lee, T., A. N. Hoofnagle, K. A. Resing, and N. G. Ahn, 2005. Hydrogen exchange solvent protection by an ATP analogue reveals conformational changes in ERK2 upon activation. J. Mol. Biol. 353:600-612.

62 22. Sours, K. M., S. C. Kwok, T. Rachidi, T. Lee, A. Ring, A. N. Hoofnagle, K. A. Resing, and N. G. Ahn, 2008. Hydrogen-exchange mass spectrometry reveals activationinduced changes in the conformational mobility of p38 $\alpha$ MAP kinase. J. Mol. Biol. 379:1075-1093.

567 23. Zhang, J., F. Zhang, D. Ebert, M. H. Cobb, and E. J. Goldsmith, 1995. Activity of the MAP kinase ERK2 is controlled by a flexible surface loop. Structure 3:299-307.

to 24. Sours, K. M., Y. Xiao, and N. G. Ahn, 2014. Extracellularregulated kinase 2 is activated by the enhancement of hinge flexibility. J. Mol. Biol. 426:1925-1935.

${ }_{573}$ 25. Xiao, Y., T. Lee, M. P. Latham, L. R. Warner, A. Tanimoto, A. Pardi, and N. G. Ahn, 2014. Phosphorylation releases constraints to domain motion in ERK2. Proc. Natl. Acad. Sci. U. S. A. 111:2506-2511.

577 26. Robinson, M. J., M. Cheng, A. Khokhlatchev, D. Ebert, N. Ahn, K.-L. Guan, B. Stein, E. Goldsmith, and M. H. Cobb, 1996. Contributions of the mitogen-activated protein (MAP) kinase backbone and phosphorylation loop to MEK specificity. J. Biol. Chem. 271:2973429739.

3з 27. Mansour, S. J., W. T. Matten, A. S. Hermann, J. M. Candia, S. Rong, K. Fukasawa, G. V. Woude, and N. G. Ahn, 1994. Transformation of mammalian cells by constitutively active MAP kinase kinase. Science 265:966-970.

887 28. Colucci-D'Amato, L., C. Perrone-Capano, and U. di Porzio, 2003. Chronic activation of ERK and neurodegenerative diseases. Bioessays 25:1085-1095.

29. Lawrence, M. C., A. Jivan, C. Shao, L. Duan, D. Goad, E. Zaganjor, J. Osborne, K. McGlynn, S. Stippec, S. Earnest, W. Chen, and M. H. Cobb, 2008. The roles of MAPKs in disease. Cell Res. 18:436.

94 30. Plotnikov, A., E. Zehorai, S. Procaccia, and R. Seger, 2011. The MAPK cascades: signaling components, nuclear roles and mechanisms of nuclear translocation. Biochim. Biophys. Acta, Mol. Cell Res. 1813:1619-1633.

1. Brunner, D., N. Oellers, J. Szabad, W. H. Biggs III, S. L. Zipursky, and E. Hafen, 1994. A gain-of-function mutation in Drosophila MAP kinase activates multiple receptor tyrosine kinase signaling pathways. Cell 76:875888.

${ }_{603}$ 32. Bott, C. M., S. G. Thorneycroft, and C. J. Marshall, 1994. The sevenmaker gain-of-function mutation in $\mathrm{p} 42$ MAP kinase leads to enhanced signalling and reduced 


\author{
M Misiura and AB Kolomeisky \\ 608 33. Oellers, N., and E. Hafen, 1996. Biochemical character- \\ sensitivity to dual specificity phosphatase action. FEBS \\ Lett. 352:201-205. \\ ization of Rolled ${ }^{\mathrm{Sem}}$, an activated form of Drosophila \\ mitogen-activated protein kinase. J. Biol. Chem. \\ 271:24939-24944.
}

${ }_{612}$ 34. Karim, F. D., and G. M. Rubin, 1999. PTP-ER, a novel

${ }_{635}$ 39. Van Kampen, N. G., 2007. Stochastic processes in physics

639 41. Camps, M., A. Nichols, C. Gillieron, B. Antonsson, nyrosine phosphatase, functions downstream of Ras1 to downregulate MAP kinase during Drosophila eye development. Mol. Cell 3:741-750.

35. Tanoue, T., M. Adachi, T. Moriguchi, and E. Nishida, 2000. A conserved docking motif in MAP kinases common to substrates, activators and regulators. Nat. Cell Biol. 2:110.

36. Zhang, J., B. Zhou, C.-F. Zheng, and Z.-Y. Zhang, 2003. A bipartite mechanism for ERK2 recognition by its cognate regulators and substrates. J. Biol. Chem. 278:2990129912.

37. Chu, Y., P. A. Solski, R. Khosravi-Far, C. J. Der, and K. Kelly, 1996. The mitogen-activated protein kinase phosphatases PAC1, MKP-1, and MKP-2 have unique substrate specificities and reduced activity in vivo toward the ERK2 sevenmaker mutation. J. Biol. Chem. 271:64976501 .

38. Bott, C. M., S. G. Thorneycroft, and C. J. Marshall, 1994. The sevenmaker gain-of-function mutation in $\mathrm{p} 42$ MAP kinase leads to enhanced signalling and reduced sensitivity to dual specificity phosphatase action. FEBS Lett. 352:201-205. and chemistry (3rd ed.). North Holland.

40. Kolomeisky, A. B., 2015. Motor Proteins and Molecular Motors. CRC Press. M. Muda, C. Chabert, U. Boschert, and S. Arkinstall, 1998. Catalytic activation of the phosphatase MKP-3 by ERK2 mitogen-activated protein kinase. Science 280:1262-1265. 


\section{${ }_{644}$ LIST OF FIGURES}

1 A simplified biochemical regulation scheme for ERK 2 considered in this work. ERK2 first must be phosphorylated by MEK (denoted as $M$ ) to become an active enzyme and to phoshorylate its substrates (denoted as $S$ ). At the same time, the phosphatase (denoted as $D$ ) can dephosphorylate ERK2 to return it to the inactive state. More details are in the text. . . . . . . . . . . . . . . . . . . . . . . . . 10

2 Catalytic constants as functions of the binding energies for Michaelis-Menten (MM, blue) and ERK2 schemes. Negative energies correspond to stronger binding. For MM scheme a monotonic behavior is observed: the stronger the interaction, the lower $k_{\text {cat }}$; while for ERK2 scheme the dependence is non-monotonic: there is an optimal value of binding energy that produces the highest $k_{\text {cat }} \ldots \ldots \ldots \ldots \ldots \ldots \ldots$

3 Michaelis constants as functions of the binding energies for Michaelis-Menten scheme (MM, blue) and ERK2 scheme (black). Negative energies correspond to stronger binding. For MM scheme the dependence is monotonic, and the stronger the binding, the lower the Michaelis constant. For ERK2 scheme the dependence is

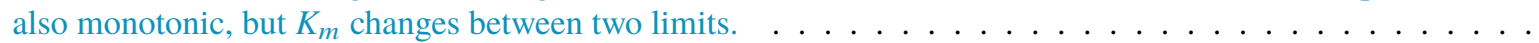

4 Specificity constants $\left(k_{\text {cat }} / K_{m}\right)$ as a functions of the binding energy for the Michaelis-Menten scheme (MM, blue) and for the ERK2 scheme (ERK2, black). Negative energies correspond to stronger binding. In the region between two vertical lines for the ERK2 scheme the decrease of strength of interactions will lead to higher values of specificity. . . . . . . . . . . . . . . . . . . . . . . . . 13

5 The specificity constant as the function of the interaction energy for varying contributions from the deactivation process. Numbers in the legend show the concentrations of the deactivator $D$ in units of moles/l. Dashed line shows the position of the maximum of the specificity constant . . . . . . . . . . . . . . .

6 Inverse catalytic turnover times, or the effective overall reaction rates for the product formation, as a function of the interaction energy for the Michaelis-Menten scheme (MM, blue) and for the ERK2 scheme (black). Concentration of substrate is $S=10^{-5}$. In the region between two vertical lines decrease of interaction energies leads to decrease of the reaction rate for MM scheme, but for the ERK2 scheme it leads to the increased turnover

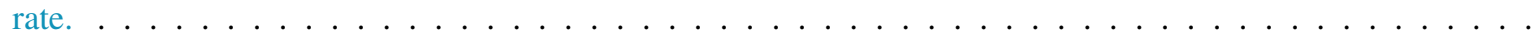


M Misiura and AB Kolomeisky

state 1

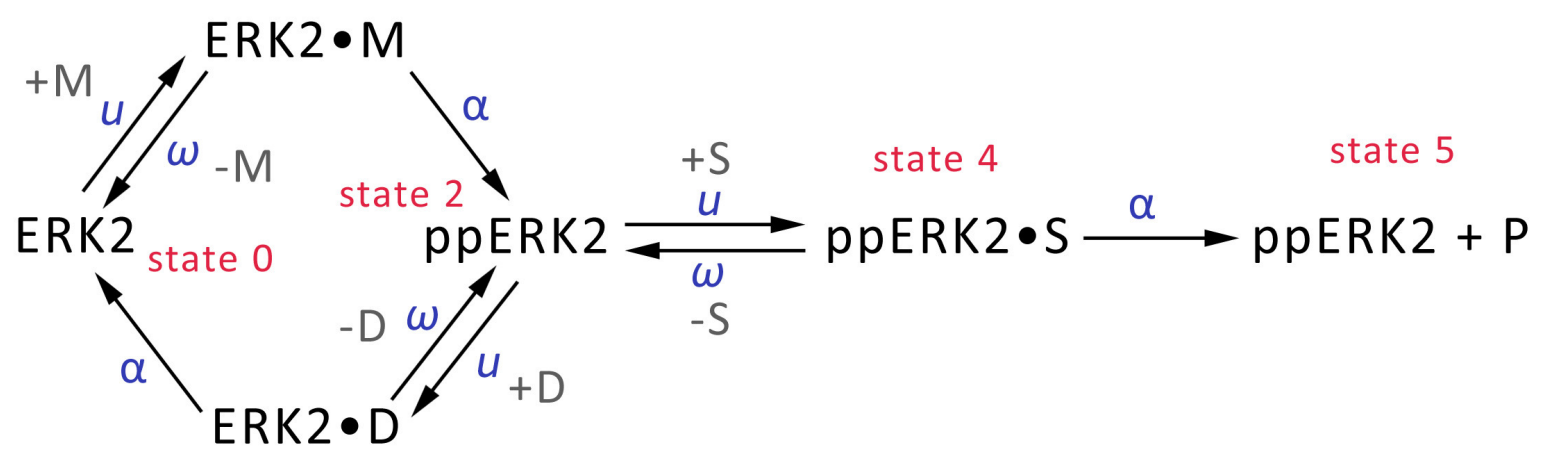

state 3

Figure 1: A simplified biochemical regulation scheme for ERK 2 considered in this work. ERK2 first must be phosphorylated by MEK (denoted as $M$ ) to become an active enzyme and to phoshorylate its substrates (denoted as $S$ ). At the same time, the phosphatase (denoted as $D$ ) can dephosphorylate ERK2 to return it to the inactive state. More details are in the text. 
bioRxiv preprint doi: https://doi.org/10.1101/409482; this version posted September 5, 2018. The copyright holder for this preprint (which was not certified by peer review) is the author/funder, who has granted bioRxiv a license to display the preprint in perpetuity. It is made available under aCC-BY-ND 4.0 International license.

Network kinetic model of ERK2 enzyme

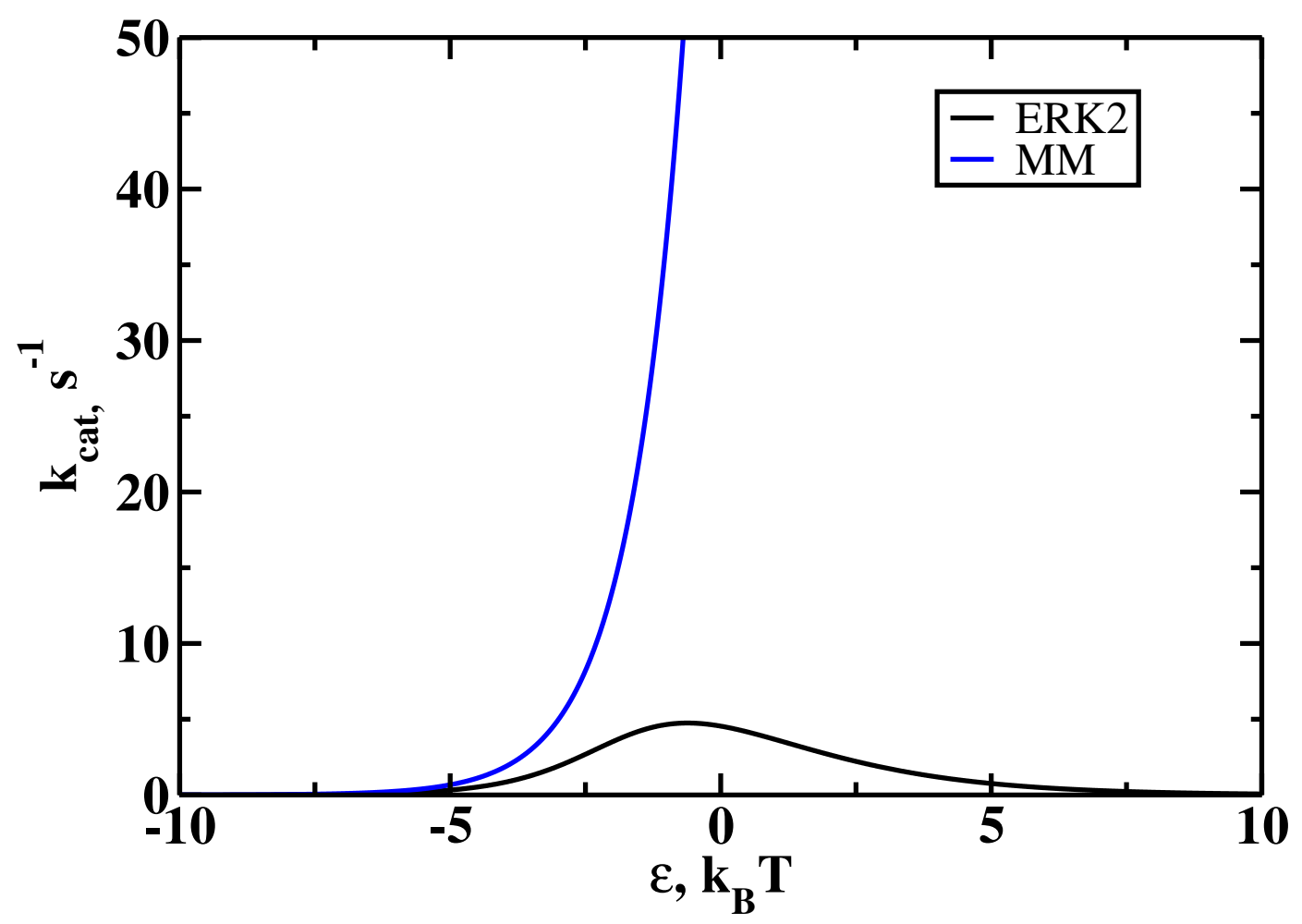

Figure 2: Catalytic constants as functions of the binding energies for Michaelis-Menten (MM, blue) and ERK2 schemes. Negative energies correspond to stronger binding. For MM scheme a monotonic behavior is observed: the stronger the interaction, the lower $k_{c a t}$; while for ERK2 scheme the dependence is non-monotonic: there is an optimal value of binding energy that produces the highest $k_{c a t}$. 
M Misiura and AB Kolomeisky

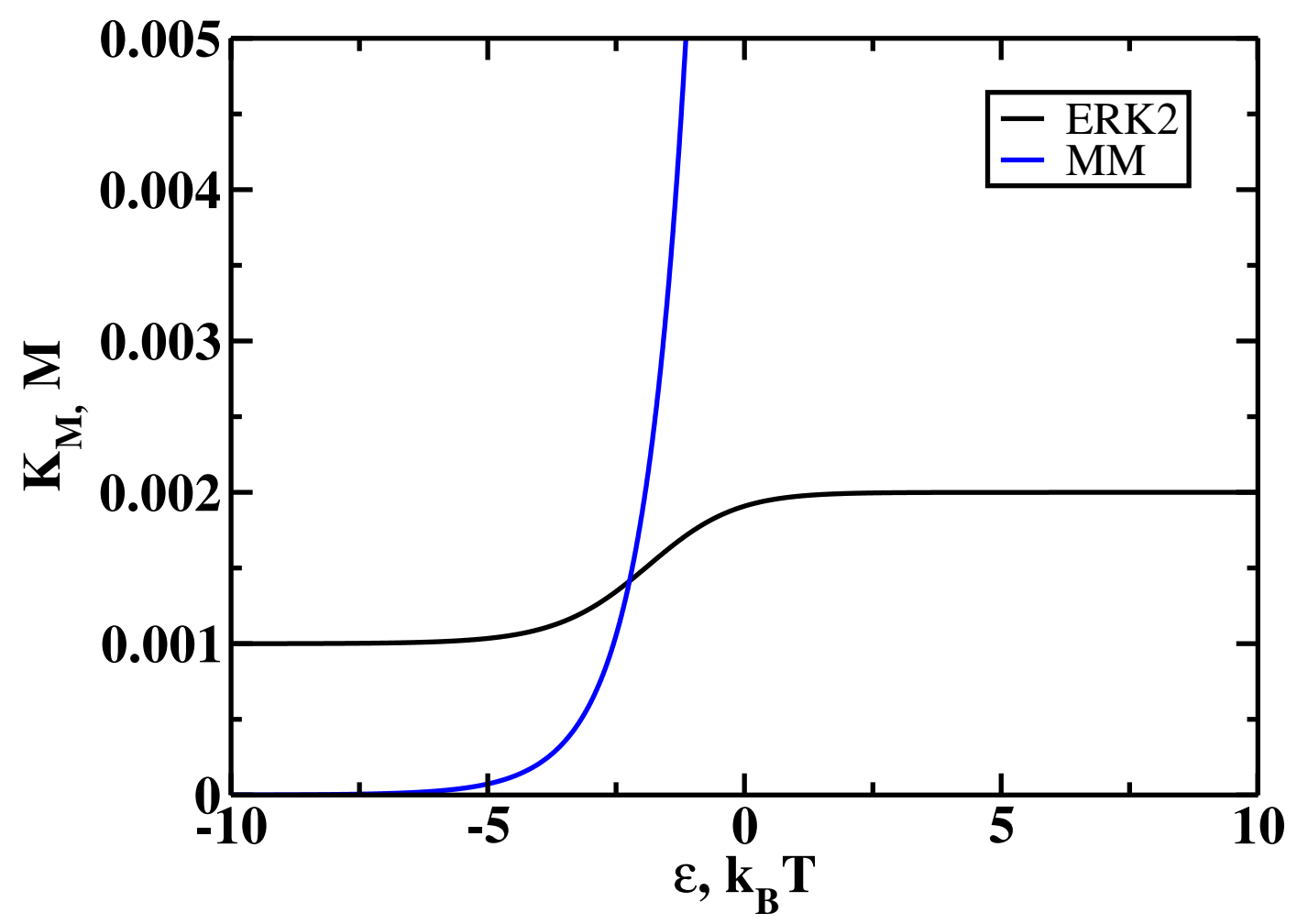

Figure 3: Michaelis constants as functions of the binding energies for Michaelis-Menten scheme (MM, blue) and ERK2 scheme (black). Negative energies correspond to stronger binding. For MM scheme the dependence is monotonic, and the stronger the binding, the lower the Michaelis constant. For ERK2 scheme the dependence is also monotonic, but $K_{m}$ changes between two limits. 
bioRxiv preprint doi: https://doi.org/10.1101/409482; this version posted September 5, 2018. The copyright holder for this preprint (which was not certified by peer review) is the author/funder, who has granted bioRxiv a license to display the preprint in perpetuity. It is made available under aCC-BY-ND 4.0 International license.

Network kinetic model of ERK2 enzyme

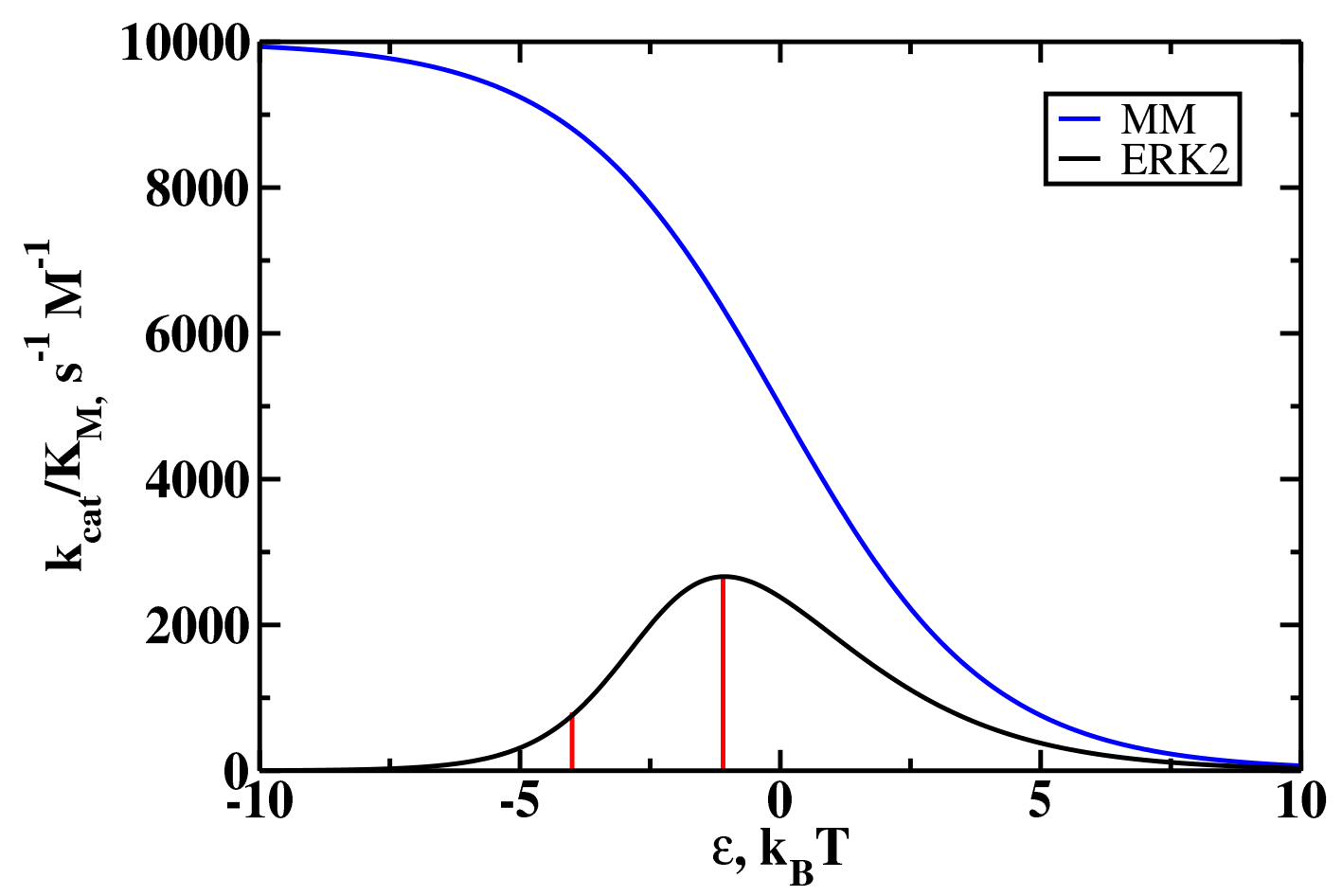

Figure 4: Specificity constants $\left(k_{c a t} / K_{m}\right)$ as a functions of the binding energy for the Michaelis-Menten scheme (MM, blue) and for the ERK2 scheme (ERK2, black). Negative energies correspond to stronger binding. In the region between two vertical lines for the ERK2 scheme the decrease of strength of interactions will lead to higher values of specificity. 
M Misiura and AB Kolomeisky

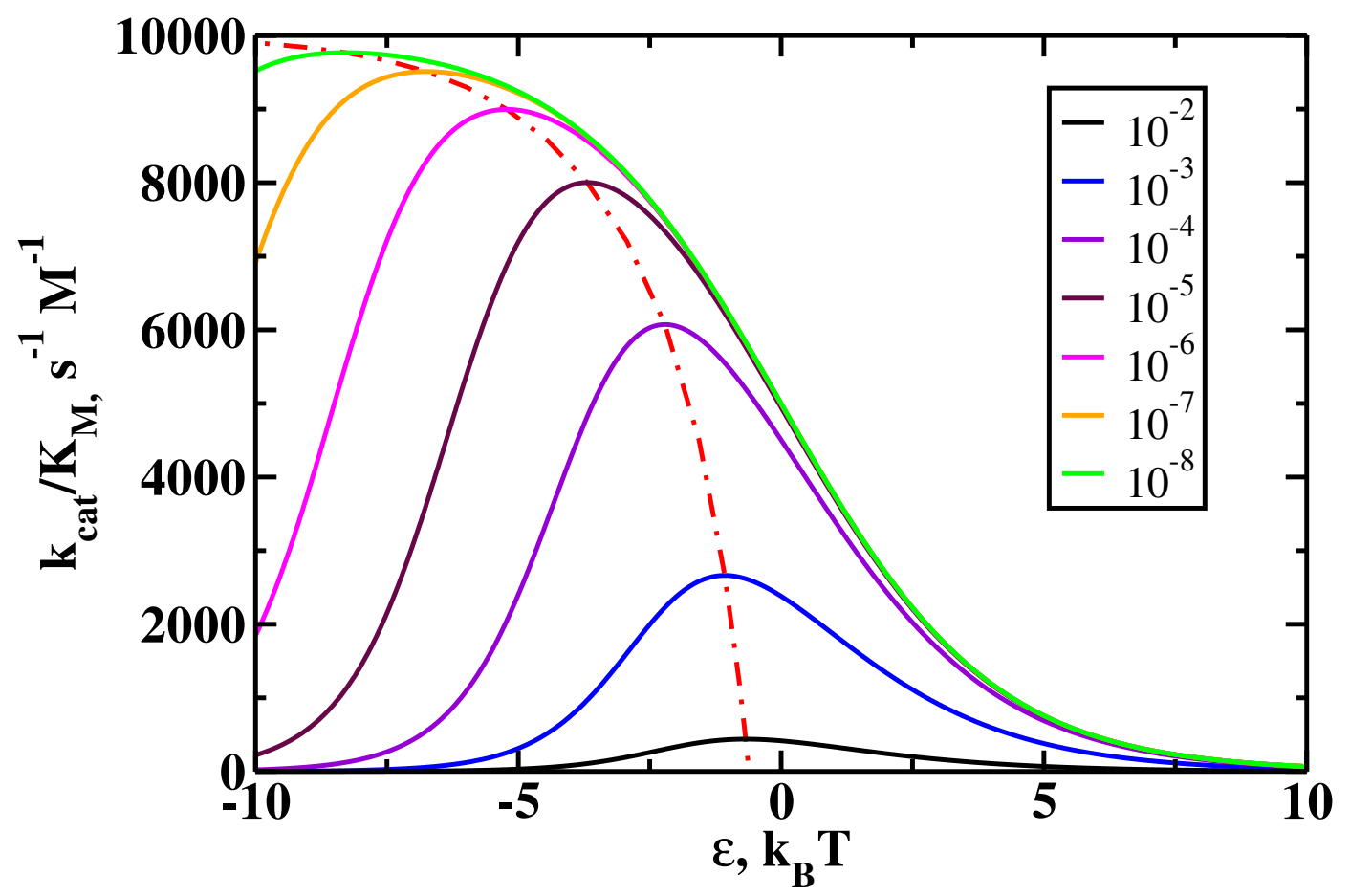

Figure 5: The specificity constant as the function of the interaction energy for varying contributions from the deactivation process. Numbers in the legend show the concentrations of the deactivator $D$ in units of moles/l. Dashed line shows the position of the maximum of the specificity constant. 
bioRxiv preprint doi: https://doi.org/10.1101/409482; this version posted September 5, 2018. The copyright holder for this preprint (which was not certified by peer review) is the author/funder, who has granted bioRxiv a license to display the preprint in perpetuity. It is made available under aCC-BY-ND 4.0 International license.

Network kinetic model of ERK2 enzyme

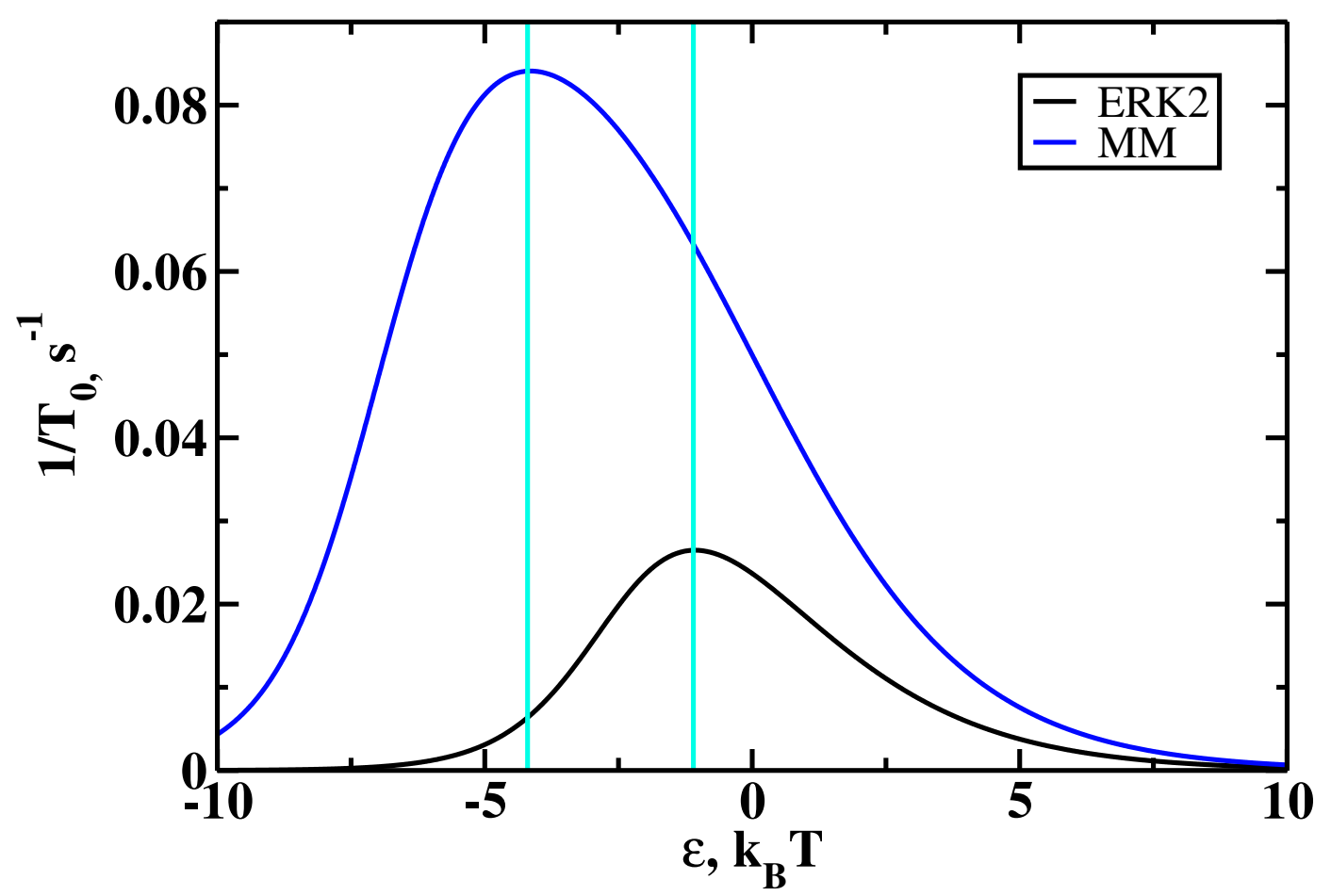

Figure 6: Inverse catalytic turnover times, or the effective overall reaction rates for the product formation, as a function of the interaction energy for the Michaelis-Menten scheme (MM, blue) and for the ERK2 scheme (black). Concentration of substrate is $S=10^{-5}$. In the region between two vertical lines decrease of interaction energies leads to decrease of the reaction rate for MM scheme, but for the ERK2 scheme it leads to the increased turnover rate. 